\title{
EFFICACY OF USING PLATELET CONCENTRATES IN THE MANAGEMENT OF HORIZONTAL RIDGE DEFECTS (EXPERIMENTAL STUDY)
}

\author{
Souzy K. Anwar ${ }^{1 *} M S$, Mona E. Lotfy ${ }^{2} P h D$, Gehan S. Kotry ${ }^{3} P h D$ and Sahar S. Karam ${ }^{4} P h D$
}

\begin{abstract} homogeneous single-moldable compound. phosphate alloplast in treating horizontal critical sized ridge defects in a canine model. sacrificed after 4 weeks. Samples were dissected and prepared for histological evaluation. of well-organized compact bone in MPM specimens versus immature woven trabecular bone in PRF ones. phosphate

1. Assistant Lecturer at Oral Medicine, Periodontology, Oral Diagnosis and Radiology Department, Faculty of

Dentistry, Alexandria University, Alexandria, Egypt.

2. Professor of Oral Medicine, Periodontology, Oral Diagnosis and Radiology Department, Faculty of Dentistry, Alexandria University, Alexandria, Egypt.

3. Assistant Professor of Oral Medicine, Periodontology, Oral Diagnosis and Radiology Department, Faculty of Dentistry, Alexandria University, Alexandria, Egypt.

4. Professor of Oral Biology, Faculty of Dentistry, Alexandria University, Alexandria, Egypt.
\end{abstract}

INTRODUCTION: The use of platelet concentrates remains controversial. Several techniques for platelet concentrates are available and each method leads to a different product with different biology and potential uses. Platelet rich fibrin (PRF) is an autologous biomaterial composed of a fibrin clot matrix entrapping leukocytes, cytokines, living progenitor cells, and platelets capable of releasing various growth factors. One of the recent platelet concentrates is the mineralized plasmatic matrix (MPM) which has a different way of preparation. It creates a stable

OBJECTIVES: The aim of this experimental study was to compare the effect of PRF to MPM when combined with biphasic calcium

MATERIALS AND METHODS: A split mouth design was carried out using the third and fourth mandibular premolars of two healthy mongrel dogs. A total of eight horizontal critical sized ridge defects were surgically created. The defects on the left side (group I); were managed with MPM composite, MPM compact layer, then collagen membrane. On the right side (group II); defects were managed with PRF mixed with biphasic calcium phosphate alloplast followed by the application of PRF compact strips, then collagen membrane. The dogs were

RESULTS: Histological results showed that quality of bone formed in MPM group was superior to PRF. This was evident with the formation

CONCLUSIONS: The fibrin network that is produced in the MPM composite allows for its homogeneity. This offers a very good stability for the graft which was found to be more effective in enhancing wound healing during guided bone regeneration of horizontal ridge defects.

KEYWORDS: Platelet Rich Fibrin, Mineralized Plasmatic Matrix, Guided bone regeneration, Horizontal ridge defects, Biphasic calcium

\section{INTRODUCTION}

The presence of an adequate bone volume of the alveolar ridge is critical for providing a predictable bony support for the gingival margin and papillae, thus contributing to an esthetic and functional final result of prosthesis (1-3).

Tissue engineering may offer a viable and attractive option besides current treatment modalities for the surgical management of severe alveolar ridge atrophy (4).

For many years, research has attempted to use biologically active molecules to achieve bone regeneration. Among these molecules are growth and differentiation factors. Growth factors are proteins which regulate the complex processes of wound healing. Growth factors play a main role on cell migration, proliferation and angiogenesis in tissue regeneration phase (5). Numerous growth factors, alone or in combination with grafting materials, have been tested for bone regeneration in animal experiments. Among these are insulin-like growth factors, fibroblast growth factors, platelet-derived growth factors, vascular endothelial growth factor, transforming growth factor- $\beta$ and bone morphogenetic proteins (6).

These growth factors are mainly located in blood plasma and platelets. The regenerative potential of platelets was introduced in 1974 by Ross et al. (7). They were amongst the pioneers who first described the extraction of growth factors from platelets. Growth factors are released after activation from the platelets trapped within fibrin matrix, and have been shown to stimulate the mitogenic response in the periosteum for bone repair during normal wound healing (8). Knighton et al (9) described the separation of patient's blood, by centrifugation, into three layers; platelet-rich plasma (PRP), platelet poor plasma (PPP) and red and white blood cell layers. It is the PRP layer that is of interest for use in wound healing since this layer contains a concentrate of the patient's platelets. These platelets have granules that contain growth factors which affect every cell as well as every tissue involved in the wound healing and regeneration of soft tissue and bone (9).

The first generation of platelet concentrate, platelet rich plasma (PRP), was introduced in dental surgery by Marx (10). However, PRP showed a weak effect regarding hard tissue regeneration (11). It also needed chemical additives as calcium chloride anticoagulant and bovine thrombin to reach the gel state. These methods were technique sensitive involving the extraction of platelet aggregates from test tube with pipetting procedure (11).

The second generation of platelet aggregation, platelet rich fibrin (PRF), was first introduced by Choukroun (12). It is a strictly autologous fibrin-rich gel produced with fresh venous blood taken from a patient's vein. The PRF protocol 
is simple, predictable and does not need biochemical additives like bovine thrombin and chemical additives as calcium chloride, to reach the gel state. So PRF is free from the concern of cross-contamination. Also, the fibrin rich gel accelerates new bone formation and soft tissue healing due to the release of growth factors $(13,14)$.

Périssé et al. (15) observed that PRP or PRF alone or in combination with bone substitute, is used to simulate an over contoured ridge volume. However, the created volume, to mimic the original ridge contour, will not be conserved and is often collapsed because it cannot support the chewing forces and muscle movements of the flap. This is due to the fact that, the PRF is not miscible seamlessly with bone substitute crystals. The mixture of PRF with inorganic filler is not possible by the prescribed conventional extraction techniques. The coagulum and inorganic compound mixture does not result in a single homogenous compound, but rather entails successive addition of products (15).

The technique of Mineralized Plasmatic Matrix (MPM) allows the improvement of the quality of bone graft / PRF mixture to create a stable homogeneous single-moldable compound that is easier to handle with additional biological properties (15).

The aim of this study was to compare and evaluate the effectiveness of PRF and MPM when mixed with biphasic calcium phosphate alloplast, in horizontal ridge augmentation.

\section{MATERIALS AND METHODS \\ Materials \\ Study Animals}

Two healthy, adult, male Mongrel (Canis familiaris) dogs, about 18-24 months old and weighing approximately 9-14 Kg. were selected for this study. The animals were adapted to the housing conditions during the study period.

\section{Grafting materials}

1. Bone substitute material (Alloplast): (Genesis BCP TM by Dio-implant, Korea) A biphasic calcium phosphate composed of Hydroxyapatite (60\%) and Beta-Tri-Calcium-Phosphate (Beta-TCP) (40\%). With $70 \%$ macropores (100 to $500 \mu \mathrm{m}$ ) and 30\% micropores $(\leq 10 \mu \mathrm{m})$.

2. Collagen Membrane: (COLLAGUIDE TM by BIOLAND, Korea) Type I atelo-collagen derived from porcine skin, (30x40x0.3mm).

3. Platelet Rich Fibrin (PRF): PRF was prepared according to Chokroun's technique (16) as follows: About $20 \mathrm{ml}$ of venous blood drawn from the cephalic vein of each dog, and immediately divided into 2 anticoagulant free glass blood collection tubes each of 10ml (SUMBOW® Plain Glass Vacuum Tube, China). Both tubes were centrifuged immediately. Blood samples were centrifuged using a tabletop centrifuge (Centrifuge Model 800 by Xiangshui. FADA medical apparatus factory, China) for $10 \mathrm{~min}$ at $3000 \mathrm{rpm}$.

After centrifugation, the resultant blood samples were divided into three layers:

o The top most layer consisted of acellular platelet poor plasma.

o The middle layer consisted of PRF or fibrin clot.

o The bottom layer consisted of red blood cells.

Each fibrin clot was gently removed from the test tube and any excess was removed by using sharp scissors. One fibrin clot was cut into small pieces and mixed with the bone graft substitute in order to fill the 2 created defects. The other fibrin clot was used to obtain the PRF membrane. The fibrin clot was gently squeezed between two sterile pieces of gauze soaked in saline thus to remove the serum and form the PRF membrane. The resultant membrane was used followed by application of collagen membrane to cover the filled defects (Figure 1).

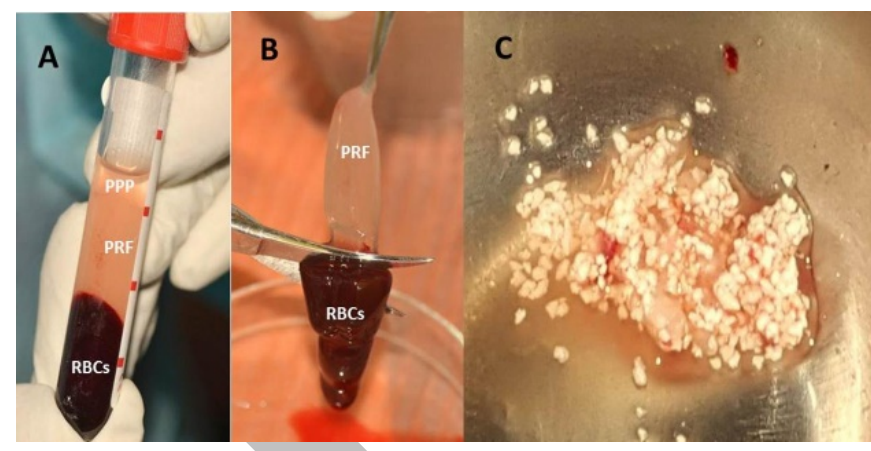

Figure 1: Clinical photographs showing PRF preparation and mixing with alloplast. (A) After centrifugation three layers are formed with PRF clot in the middle portion.(B) PRF clot separation,(C) PRF mixing with Alloplast.

4. Mineralized Plasmatic Matrix (MPM): (17) MPM was prepared as follows: About $20 \mathrm{ml}$ of venous blood drawn from the cephalic vein of each dog, and immediately divided into two $10 \mathrm{ml}$ anticoagulant free plastic blood collection tubes (VACUETTE $®$ Z No Additive by Greiner Bio-One $\mathrm{GmbH}$, Austria) each of $10 \mathrm{ml}$. Both tubes were centrifuged immediately. Blood samples were centrifuged using a tabletop centrifuge for $15 \mathrm{~min}$ at $2500 \mathrm{rpm}$.

After centrifugation, the resultant blood samples were divided into two layers:

o First layer the red blood cells in the bottom of the tube

o Second layer was in the upper portion of the tube, an amount of clear yellow plasma rich in leukocytes, platelets, mesenchymal stem cells and fibrinogen.

o The obtained liquid yellow plasma on the top of the tube was collected using a syringe, and added to a sterile cup that contained the alloplast together with a drop of dog's blood from the surgical site to provide thrombin. The latter initiated the conversion of soluble fibrinogen into insoluble fibrin under the action of calcium contained in the bone grafting material and they were all mixed together.

After a couple of minutes, a homogenous mixture of fibrin network with integrated bone graft particles inside was formed and the MPM composite was obtained. The remaining part of the collected plasma was injected over the formed MPM composite to form the MPM compact layer over it. These were placed under the collagen membrane that covered the filled defects in the study side (left side) (Figure 2). 


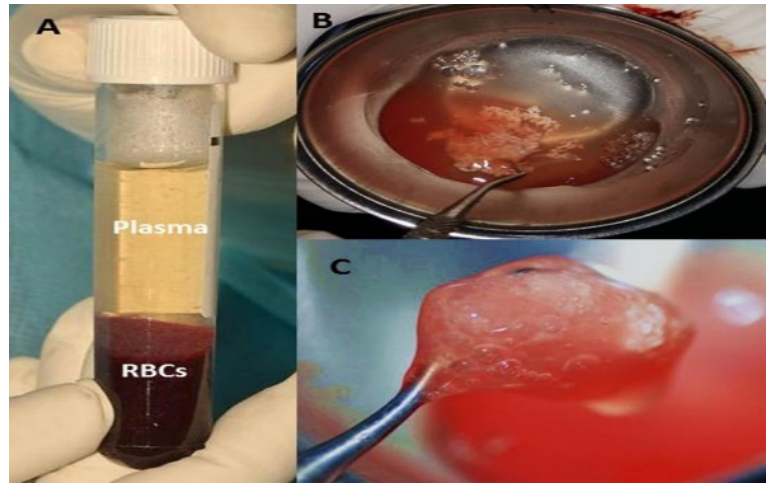

Figure 2: Clinical photographs showing MPM preparation and mixing with alloplast. (A) After centrifugation two layers are formed with clear liquid yellow plasma on the top. (B) Mixing Alloplast with the collected plasma. (C) The resultant homogenous mixture of MPM composite.

\section{Methods}

Experimental study: The study was conducted following the ethical guidelines for conduct of research on experimental animals, by the Faculty of Dentistry, Alexandria University (IRB NO:00010556 - IORG 0008839).

Study design: A split mouth comparative study design was conducted. The mandibular third (P3) and fourth (P4) premolars of the dogs were assigned as experimental and control teeth for this study.

The study included two groups as follows:

- Group I (MPM Group): included 4 surgically created horizontal well-contained critical sized ridge defects on the left side (experimental side). This group was managed with GBR using MPM composite and covered with MPM compact layer followed by the application of collagen membrane.

- Group II (PRF Group): included 4 surgically created horizontal well-contained critical sized ridge defects on the right side (control side). This group was managed with GBR using PRF cuts mixed with biphasic bone graft and covered with PRF compact strips followed by the application of collagen membrane.

Surgical procedures: All surgical procedures were performed under general anesthesia using intramuscular injection of $0.1 \mathrm{ml}$ ketamine hydrochloride (Ketamine10\%® by Alfasan, Holland.) and $0.05 \mathrm{ml}$ xylazine hydrochloride (Xyla-ject ${ }^{\circledR}$ by Adwia co. S.A.E., Egypt.), for each 100mg body weight.

\section{Surgical creation of the defects:}

- Sulcular incisions were done, followed by the reflection of mucoperiosteal flaps buccal to the mandibular third (P3) and fourth (P4) premolars in each jaw quadrant.

- Premolar teeth were extracted by sectioning the tooth with a manual saw or diamond disc, then each half was extracted separately by forceps, or teeth were extracted as one unit, when possible.

- The whole buccal walls of the sockets were removed ,with lingual walls preserved, to create two horizontal well-contained critical sized ridge defects per jaw quadrant, each about $7 \mathrm{~mm}$ mesio-distally x $8 \mathrm{~mm}$ apicocoronally x $5 \mathrm{~mm}$ bucco-lingually (18).

- On the right side, the created defects were treated with alloplast mixed with serum exudates and PRF cuts. Using
PRF compact strips and collagen membrane to cover the defects (Figure 3).

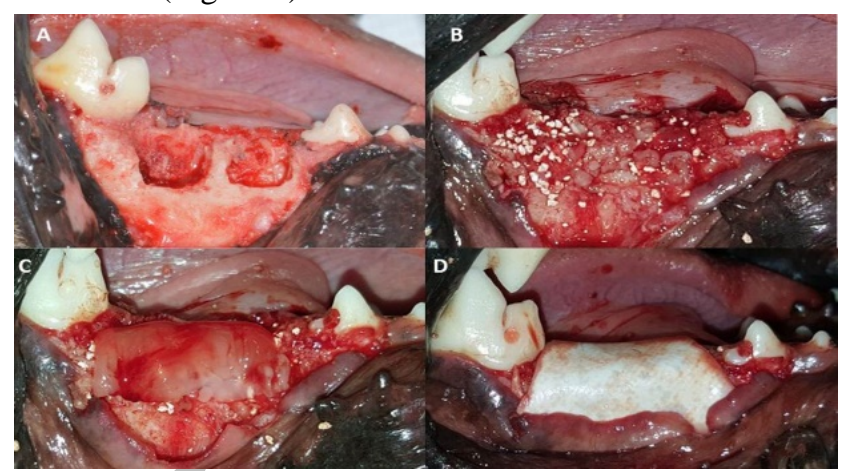

Figure 3: Clinical photographs showing: (A) The created horizontal ridge defects. (B) Management of the control side with Alloplast mixed with PRF cuts and serum exudates. (C) Defect covered with PRF membrane. (D) Collagen membrane placement to completely cover the defect.

In the experimental side (left side), the defects were treated with MPM composite. Using MPM compact layer and collagen membrane to cover the defects. (Figure 4).

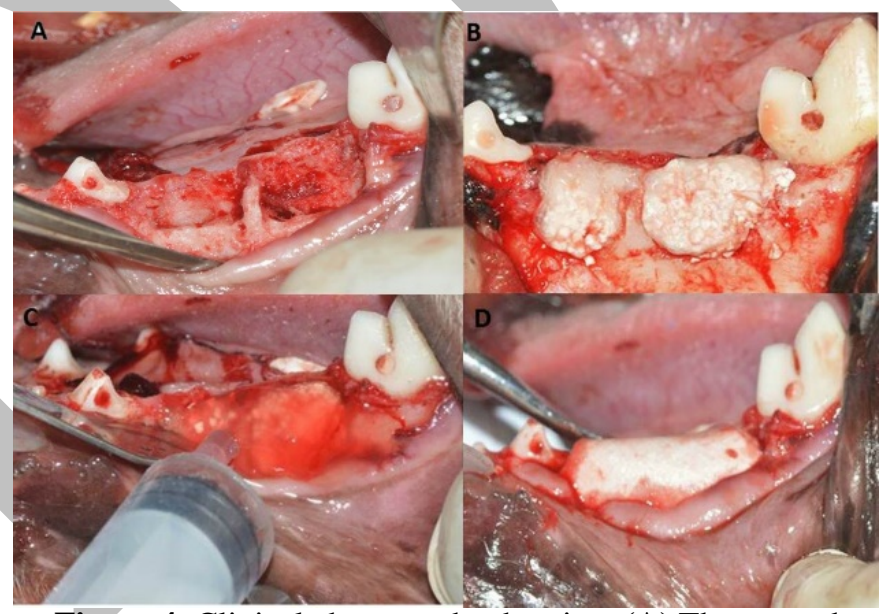

Figure 4: Clinical photographs showing: (A) The created horizontal ridge defects. (B) Management of the study side with MPM composite. (C) Part of the collected plasma injected over the formed MPM composite to form the MPM compact layer over it. (D) Collagen membrane placement to completely cover the defect

- The flaps were advanced to their original positions to cover the membranes completely. Using tension-free wound closure; periosteal releasing incisions were made to insure a tension free flap and sutured using simple interrupted sutures by 2-zero silk sutures (Ethicon silk suture, Johnson \& Jhonson, USA).

\section{Postoperative Care}

The animals received:

- Appropriate antibiotic (Ampicillin, Eipico, Egypt) that were given intramuscularly in the first day, and then mixed with dogs' food for seven days.

- Analgesics (Brufen 600 mg Abbot GmbH, Germany) given intravenously in the first day.

- Dogs were fed on soft diet in the postoperative period to reduce the possibility of local trauma to the operating sites.

Euthanization of animals and histological procedures 
Dogs were sacrificed after 4 weeks. They were euthanized with an intra-cardiac injection of xylazine+ ketamine. Following successful euthanasia, the carcass was decapitated and the lower jaws dissected out, immersed in $10 \%$ neutral buffered formalin for one day. Segments containing the operated teeth were dissected out, left in the same percentage of neutral formalin (10\%) for 4 days. They were then processed and prepared to obtain 5 microns thick bucco-lingual sections. Finally, these sections were stained with Harris Hematoxylin and Eosin Stain (H\&E) to be examined with the light microscope for general examination and evaluation of healing.

\section{RESULTS}

\section{Four weeks postoperatively} Group I (MPM group)

The histological observations of the regenerative features in this group appeared more pronounced than those in PRF group, with more defect fill with bone, greater bone maturity and better cell organization. Also, the line between the formed bone and old one could be hardly observed because of close staining level which appears almost homogenous. Interestingly, dark stained areas of immature recently formed bone trabeculae were observed merging with lightly stained mature old segments of trabeculae. This appearance reflected the close calcification level between old and new bone indicating the highly mineralized quality of newly formed bone.

The defect appeared to be replaced by thick, dense bone trabeculae covered by periosteum and thick collagen membrane on the surface. The recently formed bone mass appeared to include variable spaces of marrow stroma accommodating rich capillary supply and numerous large osteocytes in between (Figure 5).

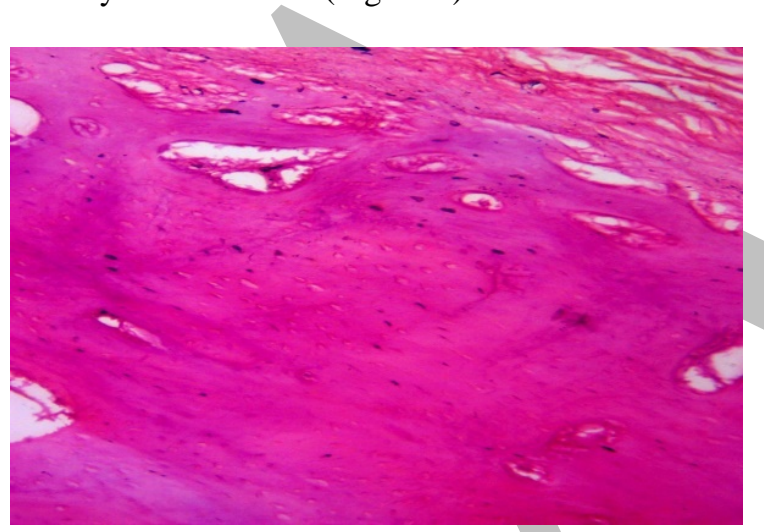

Figure (5): Photomicrograph (MPM group, one month) showing thick compact new bone formation around blood vessels, covered by periosteum and thick collagen membrane on its surface. Osteocytes in different levels indicate the maturity of osteons. H\&E x100.

\section{Group II (PRF group)}

in PRF group regeneration took place by the formation of new bone trabeculae in most of the sections. These appeared in the form of irregular, immature woven bone that was not well organized, starting to take the arrangement of primary osteons. The mass of the newly formed bone was widely spaced by numerous blood vessels and extensive areas of dense woven fibrous tissue exhibiting an interesting organization of new trabeculae continuous with the already formed ones.
Adjacent to these cellular densities, irregular spaces containing hyaline amorphous material, outlined by dark stained lines of osteoid tissue, could be traced. Most probably this hyaline amorphous material is the bone grafting content of the defect or particles remnants. Interestingly, in many sections this material appeared enclosed between sheets of densely proliferating cells (Figure 6).

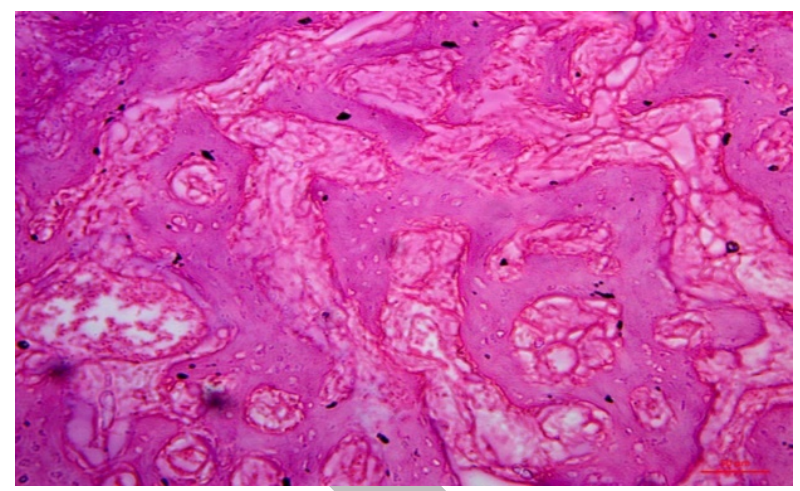

Figure (6): Photomicrograph (PRF group, one month) showing high connective tissue stroma replaced by early developed, not organized, immature woven bone. H\&E stain, $\mathrm{x} 100$.

\section{DISCUSSION}

A number of current procedures provide different approaches to the correction of horizontal alveolar ridge deficiencies. Recent systematic reviews emphasize the lack of predictable outcomes and frequent complications associated with current therapies for horizontal ridge deficiencies (19). In an effort to reduce the complication rates, especially those related to donor surgery associated with autogenous grafts, as well as to improve efficacy of outcomes, tissue engineering may offer an attractive alternative to current procedures.

In the current study, eight well-contained critical sized horizontal ridge defects were created in 2 mongrel dogs. A split mouth design was carried out in which the defects of the control side of each dog were augmented with a mixture of PRF cuts and biphasic calcium phosphate alloplast covered with a PRF membrane then completely covered with a collagen membrane. In the study side of the same dog the defects were augmented with MPM composite covered with a MPM membrane then completely covered with a collagen membrane. After one month, tissue blocks containing the studied defects with the surrounding soft tissue were obtained and examined histologically.

In both groups; the platelet concentrate forms were mixed with biphasic calcium phosphate alloplast particles to be applied inside the created defects then covered by their membrane followed by the application of collagen membrane to completely cover the augmented defect. This technique of application was also carried out by Bölükbaşı et al. (20) to maximize the contact between the defect walls and PRF from inside and from outside in order to maximize the effect of PRF. Although Bölükbaşı et al. (20) did not cover their created defects with collagen membranes we utilized collagen membranes for space maintenance to standardize the study approach, so any histological changes observed will be attributed mainly to the role of the applied material only. 
An important consideration during the use of PRF membrane was that it must be applied as early as possible after its separation and formation to ensure the maximum release of growth factors to the surgical site (21) and the produced exudate after the squeezing of the membrane can be used to hydrate the grafting material as it can be an additional source of growth factors, vitronectin and fibronectin (22).

One clinical advantage of PRF material that appeared during this study was its adhesive property. This kept the particles of alloplast together attaching them tightly to the walls of the defect, also the PRF membrane placed was adherent to the collagen membrane. This adherence is thought to provide strong stabilization of the membrane which is a prerequisite for successful GBR procedures for the prevention of downward growth of the epithelium and space maintenance. Similar to our findings Yilmaz et al. (23) and Kökdere et al; (24) observed that PRF accelerates the healing effect by keeping the particles of bone substitute and autogenous bone graft, respectively, together and attaching them tightly to the walls of the cavity by their adhesive properties favoring regeneration.

Wound healing is a staged process which involves the activity of leukocytes and platelets. For this process to work efficiently, the platelets play a vital role. Platelets are nucleate cytoplasmic fragments containing spherical or oval granules with diameters ranging from 200 to 500nm.They form intracellular storage pools of proteins including platelet-derived growth factor (PDGF), transforming growth factor (TGF), vascular endothelial growth factors (VEGF) and insulin-like growth factor (IGF) and others. Secretion of these proteins happens after the fusion of the granules with the platelet cell membrane after platelet activation; these proteins later bind to the transmembrane receptors of the target cells causing the repair or regeneration mechanisms to start (25). Thus, it is believed that platelet concentrates can aid in a more predictable outcome when added to graft materials during various regenerative procedures (25).

Platelet-rich-fibrin (PRF) is one such material that holds on to these growth factors enmeshed in the fibrin network resulting in their sustained release over a period of time that can accelerate the wound healing process. With this knowledge, research has been carried out for past few years for the clinical application of PRF. Various platelet concentrates have been studied including platelet-rich plasma (PRP). However, the short duration of cytokine release and its poor mechanical properties have resulted in the search of a new material with adequate properties for clinical application and ease of preparation. PRF has found a place in the regenerative field owing to its advantages over PRP (26).

MPM is a natural evolution of PRP and PRF. Both MPM and PRF have similar properties because their production processes (one centrifugation step, no need for additives, and use of blood collection tubes devoid of silica) are identical. Moreover, both PRF and MPM are platelet concentrates of growth factors that enhance bone and soft tissue healing. However, the later allows improvement of the mixture quality of bone graft / PRF creating a stable homogeneous single-moldable compound which can serve as an easy to handle filler with additional biological properties. Homogeneity of MPM offers stability and resistance to chewing forces. In fact the use of PRP or PRF alone will not conserve the created volume, because they cannot withstand the chewing forces and muscle movements of the flap (15).

In this regard; the histological results in this study, after one month, revealed more pronounced bone regenerative potential of MPM than PRF. The newly formed bone mass appeared more mature with better cell organization in wellformed osteons. It also included variable spaces of marrow stroma accommodating rich capillary supply and numerous large osteocytes in between. On the other hand; the bone formed in the defects of PRF group was immature woven bone accommodating multiple large fibrotic marrow spaces between trabeculae. In agreement with Périssé et al. (15) who found that the homogeneity of the MPM composite improves the performance, penetration of biological factors and qualities of the mixture at the filling site. This offers the establishment of a composite with more efficient properties than a heterogeneous compound formed of bone and PRF.

In contemporary dentistry, the quality and quantity of the bone is very important in defective ridge as it leads to incorrect placement of dental appliances like endosseous implants and inappropriate prosthetic fabrication with poor aesthetic outcome (27). In the current study, MPM specimens presented with a mass of mature compact bone. Therefore, MPM composites could act as predictable platelets concentrate regenerative biomaterials for replacing hard buccal bone in the esthetic zone, especially when immediate implants are indicated. Huynh-Ba and colleagues (28) reported that post-extraction alveolar resorption is significantly more on the buccal surfaces of the alveolar bone than the labial surfaces. In their study, they observed that it may be due to the labial anatomy of the alveolar bone which is thin and knife-edged. They also found out that the thin buccal cortical bone undergoes average resorption up to $0.8 \mathrm{~mm}$ in the anterior teeth and 1.1 $\mathrm{mm}$ in the premolar sites leading to unfavorable conditions for implant placement. As such in compensating bone loss in the esthetic zone, MPM could be the best option because it has the potential of forming a harder and mature bone that can favor the insertion of implants with good predictability and primary stability.

In the present study, MPM showed a superior effect over PRF on promoting the regenerative process in GBR procedures. It resulted in a more mature bone quality in a shorter period of time.

\section{CONCLUSION}

Within the limitations of this study we can conclude that the use of bone substitute combined with platelet concentrate in the form of MPM has shown a better histologic evidence of bone formation after one month than the use of PRF in guided bone regeneration procedures for horizontal ridge defects. This can be attributed to the homogeneity of the MPM composite which improves the performance, penetration of biological factors and qualities of the mixture at the filling site.

\section{CONFLICT OF INTEREST}

The authors declare that they have no conflicts of interest. 


\section{REFERENCES}

1. Palacci P, Ericsson I. Esthetic Implant Dentistry: Soft and Hard Tissue Management. Chicago: Quintessence; 2001. 89-100.

2. Belser UC, Schmid B, Higginbottom F, Buser D. Outcome analysis of implant restorations located in the anterior maxilla: a review of the recent literature. Int $\mathrm{J}$ Oral Maxillofac Implants. 2004;19:30-42.

3. Grunder U, Gracis S, Capelli M. Influence of the 3-D boneto-implant relationship on esthetics. Int $\mathrm{J}$ Periodontics Restorative Dent. 2005;25:113-9.

4. McAllister BS, Haghighat K. Bone augmentation techniques. J Periodontol. 2007;78:377-96.

5. Clark RA. Fibrin and wound healing. Ann N Y Acad Sci. 2001;936:355-67.

6. Trombelli L, Farina R. Clinical outcomes with bioactive agents alone or in combination with grafting or guided tissue regeneration. J Clin Periodontol. 2008;35:117-35.

7. Ross R, Glomset J, Kariya B, Harker L. A plateletdependent serum factor that stimulates the proliferation of arterial smooth muscle cells in vitro. Proc Natl Acad Sci. 1974;71:1207-10.

8. Gassling V, Douglas T, Warnke PH, Açil Y, Wiltfang J, Becker ST. Platelet-rich fibrin membranes as scaffolds for periosteal tissue engineering. Clin Oral Implants Res. 2010;21:543-9.

9. Knighton DR, Hunt TK, Thakral K, Goodson 3rd W. Role of platelets and fibrin in the healing sequence: an in vivo study of angiogenesis and collagen synthesis. Ann Surg. 1982;196:379.

10. Marx RE, Carlson ER, Eichstaedt RM, Schimmele SR, Strauss JE, Georgeff KR. Platelet-rich plasma: growth factor enhancement for bone grafts. Oral Surg Oral Med Oral Pathol Oral Radiol Endod. 1998;85:638-46.

11.Plachokova AS, Nikolidakis D, Mulder J, Jansen JA, Creugers $\mathrm{NH}$. Effect of platelet-rich plasma on bone regeneration in dentistry: a systematic review. Clin Oral Implants Res. 2008;19:539-45.

12. Choukroun J, Adda F, Schoeffler C, Vervelle A. Une opportunité en paro-implantologie: le PRF. Implantodontie. 2001;42:e62. [text in French]

13. Choukroun J, Diss A, Simonpieri A, Girard MO, Schoeffler C, Dohan SL, et al. Platelet-rich fibrin (PRF): a secondgeneration platelet concentrate. Part IV: clinical effects on tissue healing. Oral Surg Oral Med Oral Pathol Oral Radiol Endod. 2006;101:e56-e60.

14. Choukroun J, Diss A, Simonpieri A, Girard MO, Schoeffler C, Dohan SL, et al. Platelet-rich fibrin (PRF): a secondgeneration platelet concentrate. Part V: histologic evaluations of PRF effects on bone allograft maturation in sinus lift. Oral Surg Oral Med Oral Pathol Oral Radiol Endod. 2006;101:299-303.

15.Périssé J. Du PRF vers les matrices plasmatiques minéralisées en implantologie. 59ème Congrès de la SFMBCB; 2012: EDP Sciences[text in French] .

16. Dohan DM, Choukroun J, Diss A, Dohan SL, Dohan AJ, Mouhyi J, et al. Platelet-rich fibrin (PRF): a secondgeneration platelet concentrate. Part I: technological concepts and evolution. Oral Surg Oral Med Oral Pathol Oral Radiol Endod. 2006;101:e37-e44.

17. Moheb ME. The Use of Growth Factors Fibrin Network to Enhance Architecture, Mechanical and Biological Aspect of the Graft Particles. I J Pre Clin Dent Res. 2014;1:41-4.
18. Sato I, Akizuki T, Oda S, Tsuchioka H, Hayashi C, Takasaki $\mathrm{A}$, et al. Histological evaluation of alveolar ridge augmentation using injectable calcium phosphate bone cement in dogs. J Oral Rehabil. 2009;36:762-9.

19. Tonetti MS, Hämmerle CH. European Workshop on Periodontology Group C. Advances in bone augmentation to enable dental implant placement: Consensus Report of the Sixth European Workshop on Periodontology. J Clin Periodontol. 2008;35:168-72.

20. Bölükbaşı N, Yeniyol S, Tekkesin MS, Altunatmaz K. The Use of Platelet-Rich Fibrin in Combination with Biphasic Calcium Phosphate in the Treatment of Bone Defects: A Histologic and Histomorphometric Study. Curr Ther Res Clin Exp. 2013;75:15-21.

21. Su CY, Kuo YP, Tseng YH, Su CH, Burnouf T. In vitro release of growth factors from platelet-rich fibrin (PRF): a proposal to optimize the clinical applications of PRF. Oral Surg Oral Med Oral Pathol Oral Radiol Endod. 2009;108:56-61.

22. Toffler M, Toscano N, Holtzclaw D, Del Corso M, Ehrenfest DD. Introducing Choukroun's platelet rich fibrin (PRF) to the reconstructive surgery. J Implant Adv Clin Dent. 2009;6:21-32.

23. Yilmaz D, Dogan N, Ozkan A, Sencimen M, Oral BE, Mutlu I. Effect of platelet rich fibrin and beta tricalcium phosphate on bone healing. A histological study in pigs. Acta Cirúrgica Brasileira. 2014;29:59-68.

24. Kökdere N, Baykul T, Findik Y. The use of platelet-rich fibrin (PRF) and PRF-mixed particulated autogenous bone graft in the treatment of bone defects: An experimental and histomorphometrical study. Dent Res J. 2015;12:418-24.

25. Prakash S, Thakur A. Platelet concentrates: Past, present and future. J Maxillofac Oral Surg. 2011;10:45-9.

26. Gupta V, Bains VK, Singh GP, Mathur A, Bains R. Regenerative potential of platelet rich fibrin in dentistry: literature review. Asian J Oral Health Allied Sci. 2011;1:238.

27. Chandna S, Kaur K, Kaur N, Manocha A. Socket augmentation. J Int Clin Dent Res Organ. 2015;7:73.

28. Huynh-Ba G, Pjetursson BE, Sanz M, Cecchinato D, Ferrus J, Lindhe J, Lang NP. Analysis of the socket bone wall dimensions in the upper maxilla in relation to immediate implant placement. Clin Oral Implants Res. 2010;21:37-42. 